\title{
Comparison of academic performance and stress levels in open book test and closed book test and perceptions of undergraduate dental students
}

\begin{abstract}
Background: Open book test is an assessment method where the student is allowed to use the text book or notebook or any reference materials to write the examination. This will decrease the need for memorizing by the students and reduces the academic stress.

Objective: The present study was undertaken to observe the effectiveness of open book test in reducing the pre-examination stress.

Materials and methods: A total of seventy male and female first BDS students were involved in the study after obtaining the written informed consent. After recruiting, the participants they were subjected to open book test on general physiology and nerve muscle physiology. Thereafter, they were subjected to closed book test. Stress levels were assessed just before they start writing the examinations. The marks obtained in the corresponding tests were considered as academic performance respectively. After completion of both the tests student perceptions were obtained.
\end{abstract}

Results: The stress levels were significantly higher in closed book test when compared with open book test $(\mathrm{P}<0.001)$. There was no significant difference in the academic performance among open book test and closed book test (Table 1). Table 2 present's student's perceptions about open book test. Majority of the students had given positive perceptions for open book test. $51.5 \%$ of students recommend open book test to include in the curriculum

Conclusion: Significantly lower stress levels were observed before open book test when compared to closed book test. A positive experience was reported by the students regarding the open book test. The study recommends further studies in this method to recommend implementation of open book test in the medical education.

Keywords: open book test, undergraduate dental students, perceptions
Volume 6 Issue 2 - 2019

\author{
Sai Sailesh Kumar Goothy,' Steve Suphal, ${ }^{2}$ \\ Tejaswini Santoshi Bandaru, ${ }^{3}$ Swathi Movva, ${ }^{4}$ \\ Ravikanth Manyam, ${ }^{5}$ Venkata Rama Raju $A^{6}$ \\ 'Assistant Professor \& HOD (I/C), Department of Physiology, \\ Vishnu Dental College, India \\ ${ }^{2}$ First Year BDS student, Vishnu Dental College, India \\ ${ }^{3}$ Second Year BDS Student, Vishnu Dental College, India \\ ${ }^{4}$ Lecturer, Department of Physiology, Vishnu Dental College, \\ India \\ ${ }^{5}$ Professor, Department of Oral Pathology,Vishnu Dental \\ College, India \\ ${ }^{6}$ Vice Principal \& Professor, Department of Prosthodontics, \\ Vishnu Dental College, India
}

\begin{abstract}
Correspondence: Sai Sailesh Kumar Goothy, Assistant Professor \& HOD (I/C), Department of Physiology,Vishnu Dental College, Bhimavaram, West Godavari District, Andhra Pradesh, India, Email dr.saisailesh@gmail.com
\end{abstract}

Received: February 20, 2019 | Published: March 29, 2019

\section{Introduction}

Medical education is emerging with introduction and implementation of new teaching, learning and assessment methods. Medical education units in the academic institutions striving to motivate the students and to convert the learning into student centered from teacher centered. Open book test is an assessment method where the student is allowed to use the text book or notebook or any reference materials to write the examination. This will decrease the need for memorizing by the students and reduces the academic stress. ${ }^{1}$ Earlier studies reported that open book test is worth implementing as it is a best assessment tool and also promote student centered learning. Further, it helps to reduce the stress levels of the students. ${ }^{2}$ Open book test also motivates the student to use the textbooks more frequently and limits the last minute preparations for the examinations. Further, open book test improves the higher-order thinking skills of the students as they critically analyze the contents in the textbook also get adequate time to present those contents in a creative way in the examination. ${ }^{3}$ It was reported that there is no difference in the marks obtained by open book test and closed book test. ${ }^{4}$ As the main goal of the assessment is to assess the thinking skills rather memorizing skills. Further, the environment is relaxed to the students. As the open book test reduces examination stress without affecting the academic performance, it can be implemented in the medical education. ${ }^{5-7}$ Recently, All India council for technical education recommended open book test for engineering curriculum. However, the studies related to implementation of open book test are relatively less. Hence, the present study was undertaken to observe the effectiveness of open book test in reducing the pre-examination stress.

\section{Materials and methods}

\section{Study participants}

A total of seventy male and female first BDS students were involved in the study after obtaining the written informed consent. Willing, apparently healthy participants were recruited for the study.

\section{Study setting}

The present study was conducted at Department of Physiology, Vishnu Dental College, Bhimavaram.

\section{Study design}

After recruiting, the participants they were subjected to open book test on general physiology and nerve muscle physiology. Thereafter, they were subjected to closed book test. Stress levels were assessed just before they start writing the examinations. The marks obtained in the corresponding tests were considered as academic performance respectively. After completion of both the tests student perceptions were obtained. 


\section{Open book test}

Students were briefed about the open book test a study hour was conducted where the students studied the general physiology and nerve muscle physiology from their text books. All the doubts were clarified in the study hour. Open book test was conducted for 30 marks in general physiology and nerve muscle physiology. Students were allowed to use their text books in the examination hall. However, assessment to online materials is not allowed. Answer scripts were evaluated on same day following the answer key which was prepared after thorough discussion with all the faculty members of physiology department.

\section{Closed book test}

Closed book test was conducted for 30 marks in general physiology and nerve muscle physiology. However, the question paper was different for open book test and closed book test. Answer scripts were evaluated on same day following the answer key which was prepared after thorough discussion with all the faculty members of Physiology department.

\section{Outcome measures}

\section{Academic performance}

Marks obtained in the open book test and closed book test was considered as academic performance respectively.

\section{Stress levels}

Stress levels were assessed using perceived stress scale. ${ }^{8}$

\section{Perception of the students}

Perception of the students was obtained using standard

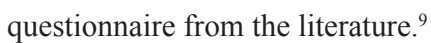

\section{Ethical considerations}

The present study was approved by institutional research committee. Informed consent was obtained from all the students.

\section{Data analysis}

Data was analyzed using SPSS 20.0 version. Data was represented as mean and SD. Student $t$ test was applied to observe the significance of difference between the groups. Perceptions of students were expressed as frequency and percentages. Probability value less than 0.05 was considered as significant.

\section{Results}

The stress levels were significantly higher in closed book test when compared with open book test $(\mathrm{P}<0.001)$. There was no significant difference in the academic performance among open book test and closed book test (Table 1). Table 2 present's student's perceptions about open book test. Majority of the students had given positive perceptions for open book test. $51.5 \%$ of students recommend open book test to include in the curriculum.

Table I Comparison of stress scores and academic performance in open book test and closed book test

\begin{tabular}{llll}
\hline Parameter & Open book test & Closed book test & P value \\
\hline $\begin{array}{l}\text { Stress scores } \\
\text { Academic }\end{array}$ & $10 \pm 3$ & $18 \pm 3$ & $0.0001^{* * *}$ \\
performance & $17 \pm 6$ & $18 \pm 4$ & 0.248
\end{tabular}

Data was presented as mean and SD. $(* \mathrm{P}<0.05$ is significant, $* * \mathrm{P}<0.01$ is significant, $* * * P<0.001$ is significant).

Table 2 Perceptions of students about open book test

\begin{tabular}{llllll}
\hline Question & S.A & A & N & D & S.D \\
& F (\%) & F (\%) & F (\%) & F (\%) & F (\%) \\
\hline Happy to sit for the test & $28(40)$ & $36(51.4)$ & $6(8.6)$ & 0 & 0 \\
Reducing the pressure of memorizing & $15(21.4)$ & $38(54.3)$ & $17(24.3)$ & 0 & 0 \\
Had a Good experience & $20(28.6)$ & $44(62.9)$ & $6(8.6)$ & 0 & 0 \\
Open book examination is suitable for course & $17(24.6)$ & $44(63.8)$ & $4(5.8)$ & $3(4.3)$ & $1(1.4)$ \\
Suitable for education & $28(40)$ & $36(51.4)$ & $4(5.7)$ & $2(2.9)$ & 0 \\
Recommend Open book test to include in the curriculum & $24(35.3)$ & $35(51.5)$ & $7(10.3)$ & $2(2.9)$ & $2(2.9)$ \\
\hline
\end{tabular}

Data presented as frequency and percentage.

\section{Discussion}

The present study was undertaken to observe the effectiveness of open book test in reducing the pre-examination stress. The stress levels were significantly higher in closed book test when compared with open book test $(\mathrm{P}<0.001)$. There was no significant difference in the academic performance among open book test and closed book test (Table 1). Table 2 present's student's perceptions about open book test. Majority of the students had given positive perceptions for open book test. $51.5 \%$ of students recommend open book test to include in the curriculum. As there are drastic changes in the students, the medical education must be reviewed and adopted to fit to the needs of the students to make the teaching more students friendly. Medical education in India was drastically developing and contributed in transforming the learning as active learning from the passive mode of learning. Open book test is a simple and student friendly assessment program which helps in relieving the stress levels in the students which is the major cause for the suicides of college students. ${ }^{10}$ It removes the hectic memorizing and recalling the subject and improves the reasoning ability of the students. ${ }^{11}$ Earlier studies reported that the academic performance was not difference between the open book test and closed book test. ${ }^{12}$ In the traditional closed book test, when considered in the point of a slow learner, he prefers to read or memorize only the important chapters and important questions which are repeated more frequently in the examinations. In this practice he will not read all the chapters so he cannot have concept or 
minimum knowledge in these areas. This is the major reason why we see majority of students can get through the traditional examinations but unable to achieve good ranks in the competitive examinations. In contrast, open book motivates the higher order thinking skills of the students as they learn reasoning, analyzing and to solve real life problems. ${ }^{13}$ The present study results supports earlier studies as we have not observed any difference in the academic performance.

The student who is regular to classes and know the concept only can justify the open book test as most of the medical and dental subjects are concept oriented. The question paper should consist of more concept based questions to fulfill the objective. As the open book test promotes the active learning and best assessment method and further decreases the stress levels, it can be implemented in the curriculum of health sciences.

There are certain misconceptions regarding open book test like medical students who need to treat the patients cannot do justice when treating as they are following open book test method for examination. This can be answered in the following way. Open book test does not mean to train student to open the text book and treat cases. When there is a transition of the assessment method from closed book test to open book test, there will be change in the questioning pattern also. Even if the student excels in the traditional method of examinations it is a fact that he/she cannot be good at practice and skilled doctor unless the student understands the case in right way, chooses the right solution and best at implementation. The open book method contains case based questions and the questions that need application of thinking skills enabling the student to pick up the right solution and present it in an appropriate way and this is possible only when the sound is sound in knowledge. It discourages the mugging up process, as it known that mugging up answers without concepts are of short-term memory processes wherein the student will not carry the concepts throughout the his carrier. The real aim behind the innovations in medical education and the transition from teacher centric to studentcentric method is to ensure the enhancement of true knowledge for implementation and practice when in field. To some extent it is believed that the open book test method may contribute for the quality outcome in the medical field.

\section{Conclusion}

Significantly lower stress levels were observed before open book test when compared to closed book test. A positive experience was reported by the students regarding the open book test. The study recommends further studies in this method to recommend implementation of open book test in the medical education.

\section{Acknowledgments}

None.

\section{Conflicts of interest}

The authors declare there is no conflict of interest.

\section{References}

1. Tor Vidar Eilertsen, Odd Valdermo. Open-book assessment: A contribution to improved learning? Studies in Educational Evaluation. 2000;26(2):91-103.

2. Richard Brightwell, Janine-Helen Daniel, Angus Stewart. Evaluation: is an open book examination easier? Bioscience education. 2004;3(1);1-10.

3. Bloom BS, Hastings JT, Madaus GF. Handbook on formative and summative evaluation of student learning. New York: McGraw-Hill; 1971:923.

4. Kalish RA. An Experimental evaluation of the open-book examination. Educ Psychol. 1958;49(4):200-204.

5. Upson RH. Open-book Examination. J Engin Educ.1953;43:429.

6. Niels Krarup, Noe Naeraa, Christian Olsen. Open-book tests in a university course. Higher Education. 1974;3(2):157-164.

7. Teodorczuk A, Fraser J, Rogers GD. Open book exams: A potential solution to the "full curriculum"? Med Teach. 2018;40(5):529-530.

8. Cohen S, Williamson G. Perceived stress in a probability sample of the United States. In: Spacapan S, Oskamp S, editors. The Social Psychology of Health. Newbury Park, CA: Sage; 1988.

9. Kruger SJ. Students' and lecturers' perceptions of the effect of open-book examinations on teaching and assessment at departments of accountancy at South African universities. South African Journal of Higher Education . 2018;32(5):66-85.

10. Sai Sailesh Kumar Gothy, Ravikanth Manyam, Venkata Rama Raju Alluri. Students suicides-can't we stop this? Journal of Clinical and Diagnostic Research. 2019;13(2):CL01.

11. Tussing L. A consideration of the open book examination. Educational and Psychological Measurement. 1951;11(4-1):597-602.

12. Hamidreza Mahmoudzadeh Sagheb, Zahra Heidari, Mahdi Mohammadi. A survey of the students' perspectives of open-book examinations in the histology/embryology course. Journal of Medical Education. 2015;14(1):27-32.

13. Boniface D. Candidates' use of notes and textbooks during an open-book examination. Educational Research. 1985;27(3):201-209. 